\title{
Development approach of a programmable and open software package for power system frequency response calculation
}

\author{
Yuzheng Xie ${ }^{1}$, Hengxu Zhang ${ }^{1}$, Changgang $\mathrm{Li}^{1 *}$ and Huadong Sun ${ }^{2}$
}

\begin{abstract}
Dynamic behaviour of frequency is crucial for power system operation and control. Several frequency response models have been proposed to reveal frequency dynamics from different aspects. A comprehensive software package incorporating major frequency response models is needed for analysis and control of power system frequency dynamics. In this paper, an approach for developing a programmable and open software package for frequency response studies is proposed. The framework of the package is extendable with reduced frequency response models. Essential models for frequency response study are included, e.g., generator, load, and under-frequency load shedding (UFLS). The provided application program interfaces (APIs) enable simulation with high-level languages by calling dynamic link library and makes the package programmable. An advanced application module is developed for quantitative assessment of transient frequency deviation. APIs can also be used for model extension and secondary development. To demonstrate the usage of the package, several examples are illustrated to explain how to perform simulations with the package, and to perform advanced applications using scripting with the provided APIs.
\end{abstract}

Keywords: Frequency response, Frequency control, Software engineering, Under-frequency load shedding, Transient frequency deviation security, Power systems

\section{Introduction}

As one of the most important electrical parameters of power systems, frequency and its dynamic characteristic is crucial for power system operation and control [1]. Frequency dynamics interacts with many devices in power systems in two ways. First, the performance of many devices are affected by frequency, e.g., speed governor of synchronous generators, induction motors, power system stabilizer (PSS) with frequency as input, and reactance and susceptance of transmission lines or shunt components. Second, the dynamic behaviour of system frequency is also affected by those frequencydependent devices. Besides, dynamic characteristic of frequency is a key factor influencing power system protection. Generators are protected against abnormal

\footnotetext{
* Correspondence: lichgang@sdu.edu.cn

'Key Laboratory of Power System Intelligent Dispatch and Control of the Ministry of Education (Shandong University), 17923 Jingshi Road, Jinan, Shandong 250061, China

Full list of author information is available at the end of the article
}

frequency deviation with over-speeding and underspeeding protective relays. Under-frequency load shedding (UFLS) is an important resort to prevent power system collapse in the event of large generation deficit $[2,3]$. The cooperation between the generating unit protective relays and UFLS is important for power system frequency stability [4]. Moreover, investigation into some blackouts indicates that large frequency deviation is a main factor pushing power systems to the edge [5]. In the process of power systems restoration, frequency deviation should be carefully restricted by gradually starting generators and loads to avoid large frequency deviation and subsequent system failure [6]. Furthermore, with large scale of renewable energy integrated into power grids, power fluctuation from renewables will lead to continuous power system frequency fluctuation and deviation [7]. Primary and secondary frequency regulation have important effect on preventing frequency deviation. The participation of renewable energy generation in frequency regulation also plays an important role in frequency security, regulation, 
and control. Therefore, it is necessary to study the dynamic behaviour of power system frequency for improving the operation performance of modern power systems.

There are two ways to obtain frequency response of power systems. One is the measurement from devices such as phasor measurement units (PMUs) [8] and digital fault recorders from high voltage levels, and frequency disturbance recorder (FDR) $[9,10]$ and PMU Lights [11] from low voltage levels. The measured frequency reveals the actual dynamic behaviour of power systems. However, without the knowledge of event type and location, system frequency behaviour can hardly be examined with the measured frequency. In most situations, power systems are operated in ambient mode with little frequency deviation. Dynamic behaviour of frequency with large frequency deviation can be rarely observed. Therefore, the frequency dynamic behaviour can hardly be studied using measurement data.

The other way to get frequency response is to perform numerical simulations with mathematical models of devices. It can easily create scenarios with large frequency deviation by setting up appropriate events. It is the most used technique for studying frequency dynamic characteristics and designing proper control strategies. Numerical simulation methods can be classified into two categories: detailed methods and reduced methods. Full time-domain simulation is the widely used detailed method and provides detailed models of the network and dynamic equipment with appropriate simplification. With coupled active power-frequency dynamics and reactive power-voltage dynamics, frequency, voltage, and angle dynamics can be studied at one time. With detailed network, full timedomain simulation can easily reveal the space-time distribution characteristics of frequency dynamics [12]. With area interconnection and integration of large numbers of devices, computational burden of full time-domain simulation is significantly increased. Thus, full time-domain simulation is not suitable for such circumstance as online security evaluation and emergency control. To supplement the study of frequency dynamics, reduced models such as average system frequency model (ASF) [13], single machine model and system frequency response model (SFR) [14] are adopted. Unlike full time-domain simulation, the reduced methods consider only the active powerfrequency dynamics to reduce computation burden.

During the long history of power systems research and operation, full time-domain simulation is extensively used in commercial power system analysis software such as PSS/E and DigSILENT Power Factory. The availability of those commercial software package greatly promotes the research and operation of modern power systems. The reduced models, however, have been developed by researchers for specific studies, and there is no comprehensive software package incorporating these reduced models. The aim of this paper is to propose a framework for developing a programmable and open software package for frequency dynamics study with reduced models.

The rest of the paper is organized as follows. A direct current power flow based frequency response model (DFR) is proposed and reduced frequency response models are reviewed in Section 2. A software package is proposed in Section 3 to incorporate major reduced frequency response models. To demonstrate the usage of the package, the IEEE 9-bus model, NPCC 140-bus model, and a 1000-bus model from China are tested in Section 4. The features of the proposed package are summarized and conclusions are drawn in Section 5.

\section{Reduced frequency response models}

\subsection{DFR model}

Full time-domain simulations can simulate frequency response of power systems in detail. However, due to the coupled active power-frequency dynamics and reactive power-voltage dynamics, both frequency and voltage need to be considered when studying power system dynamic behaviour. The influence of frequency and voltage can hardly be distinguished. Consequently, the DFR model is proposed in this paper to decouple frequency and voltage dynamics and to consider the influence of the network. In the DFR model, system network is simulated by direct current power flow so the redistribution of imbalanced power between different generators and the space-time distribution characteristics of frequency can be considered. To focus on active power-frequency dynamics, some assumptions are made as follows. (1) Excitation and regulation system is strong enough to hold the generator terminal voltage and thus, the dynamics of the excitation and regulation systems and the PSS can be eliminated for its negligible influence on active power-frequency dynamics. (2) Generators swing equations are reserved while the influence of transient process of the internal windings on system frequency change can be neglected due to the constant generator terminal voltage. Since turbine-governors have significant effect on power system frequency dynamics, details of the turbine-governor are modelled in the DFR model.

In the DFR model, the dynamic behaviour of frequency is only influenced by active power change. With constant bus voltages, direct current power flow is introduced to simulate the network when calculating active power flow under initial operating condition [15]:

$$
\mathbf{P}=\mathbf{B} \boldsymbol{\theta}
$$

where $\mathbf{P}$ is the active power injection, $\boldsymbol{\theta}$ is the voltage angle of all buses except the slack bus, and $\mathbf{B}$ is the network susceptance matrix. 
The assumption of constant voltage leads to the simplification of load models. With constant terminal voltage, reactive power of loads can be ignored and the polynomial load model [16] can be reduced as a static active power load with frequency dependency:

$$
P_{L}=P_{0}\left(1+K_{p f} \Delta f\right)
$$

where $P_{L}$ is the actual load, $P_{0}$ is active power of the load under initial condition, $K_{p f}$ is the load regulation coefficient, and $\Delta f$ is frequency deviation.

Other models can also be simplified with appropriate assumptions. For example, high voltage direct current links (HVDC) can be represented as loads for sending and receiving ends with or without frequency dependency.

With the simplifications of the generating units, network, loads and other equipment, the DFR model can be shown in Fig. 1. Quantities in Fig. 1 are listed as follows. $\omega_{i}, \delta_{i}, P_{m i}$, and $P_{e i}$ are rotor speed, rotor angle, mechanical power, and electrical power of generating unit $i . \Delta f_{j}$ and $P_{j}$ are the bus frequency and active power of load $j$.

Similar to full time-domain simulation, the DFR model can be expressed in terms of differential-algebraic equations (DAEs), and can be solved by step-by-step integration such as implicit trapezoid integration. Comparing with full time-domain simulation, the computational burden of the DFR model is greatly reduced and it achieves a better computational efficiency with acceptable accuracy. The DFR model can be used to analyse events of load change, generator tripping, etc. It can be also applied to fast frequency response calculation for active power disturbances and event screening.

With the introduction of direct current power flow, the DFR model is applicable to systems in which the network reactance is significantly greater than the resistance, e.g., high voltage transmission systems. The DFR model is primarily useful for cases where frequency stability is the main concern and angle stability and voltage stability can be maintained.

\subsection{ASF model}

In real systems, the frequency difference among buses is trivial if generators remain in synchronism during transient process [13]. Thus frequency at different buses can be treated as uniform and space-time distribution of frequency can be neglected. By neglecting the network, the DFR model reduces to ASF model from which uniform frequency can be achieved. The general diagram of the ASF model is shown in Fig. 2(a) where turbine-governors and loads are modelled explicitly. $P_{m \Sigma}$ and $P_{e \Sigma}$ are total mechanical power and total active power load of the system. $\Delta \omega$ is the uniform frequency of the system which is generated from the equivalent swing equation. In addition, all loads can be aggregated into an equivalent load model to simplify the ASF model. It can be applied in applications such as spinning reserve allocation, load frequency control, etc $[17,18]$.

The ASF model can be modelled with DAEs and solved with step-by-step integration. With network neglected, the computational burden of the ASF model is much less than that of the DFR model.

\subsection{Single machine model}

Single machine model can be treated as a special case of the ASF model, as shown in Fig. 2(b). It is obtained by further aggregating all turbine-governors and loads in the ASF model. The nonlinearity of the turbinegovernors, such as the valve limits and dead bands, is reserved. The structure of aggregated turbine-governors is usually the same as normal turbine-governors. For example, for stand-alone system with most of electricity generated by thermal generating units, steam turbinegovernor is preferred for the aggregated model. Stepby-step integration is also used to solve the nonlinear single machine model.

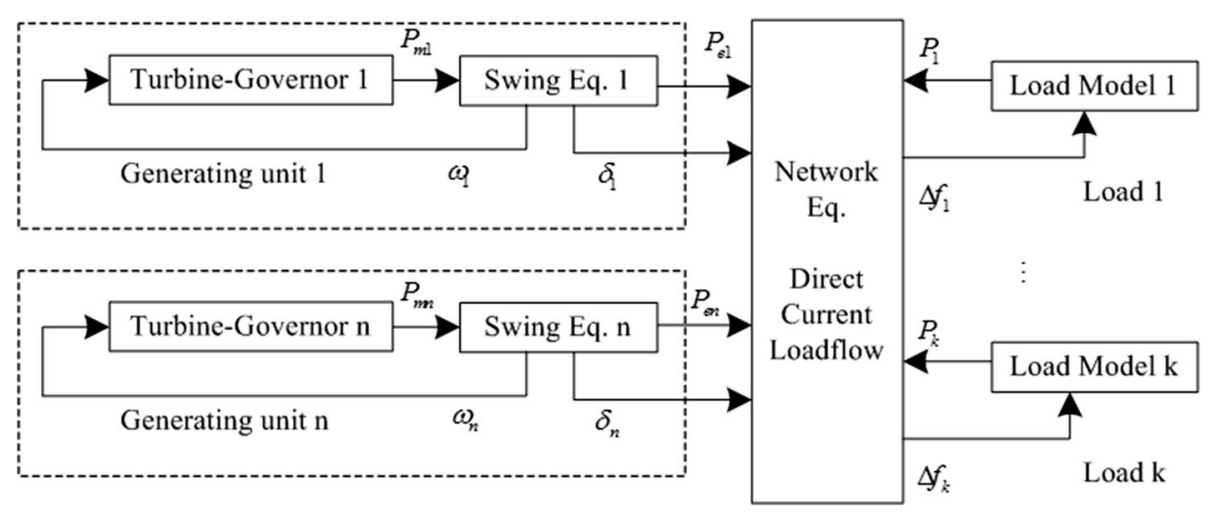

Fig. 1 Schematic diagram of DFR model 


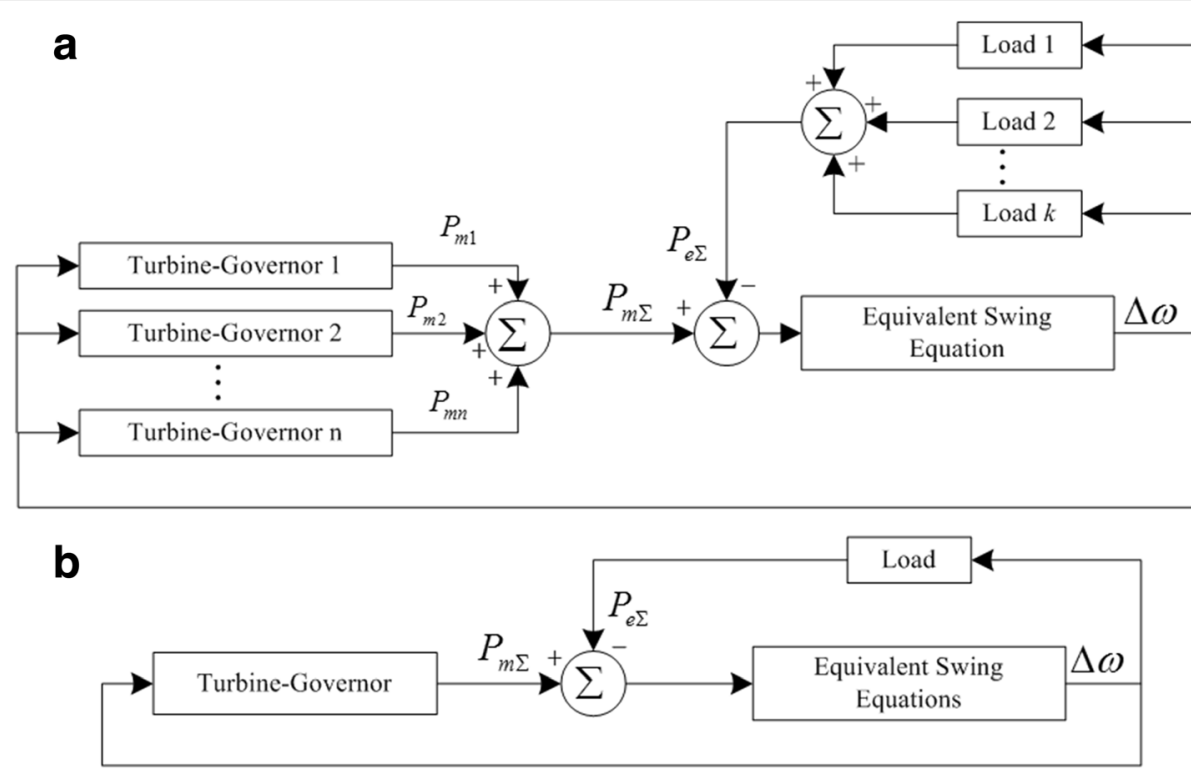

Fig. 2 Block diagram of ASF and single machine model. a General ASF model. b Single machine model

\subsection{SFR model}

Nonlinearity of the turbine-governors is considered in the DFR model, ASF model and the single machine model. No analytical expression can be directly obtained and step-by-step integration is the most popular method to get discrete response. By neglecting the nonlinear blocks and small time constants, SFR model was proposed in [14] to derive an analytical expression of frequency dynamics for stand-alone systems, in which the generators are dominated by reheat steam turbines. The block diagram of the SFR model is shown in Fig. 3 where $P_{d}, P_{m}, H, D, R, F_{H}, T_{R}$, and $K_{m}$ are disturbance, mechanical power, inertia, damping, droop, fraction of total power generated by high-pressure turbine, time constant of reheater, and mechanical power gain factor of the aggregated system. Using the analytical expression given in [14], the largest frequency deviation, its corresponding time, and steady frequency under a given active power disturbance can be calculated. Several research adopts SFR model for adjusting UFLS $[19,20]$.

\subsection{Discussion}

The frequency dynamic characteristics can be categorized in different ways. For applications depending on the overall dynamic characteristics of frequency, e.g., frequency regulation, uniform frequency is usually assumed and the frequency at different locations is treated as the same. In this case, network can be neglected, and ASF model, single machine model, and SFR model are appropriate. The space-time distribution feature of frequency during event is of most interest for applications such as event location and oscillation detection where the difference between the generators at different locations should be taken into account. In this case, the influence of network should be retained to get the space-time characteristics, and the DFR model is suitable for such applications.

For detailed study of power system dynamic characteristics, the coupling between active power-frequency dynamics and reactive power-voltage dynamics should be included, resulting in the complex full time-domain simulation. However, for cases where frequency dynamic characteristic is of most concern and voltage dynamic is

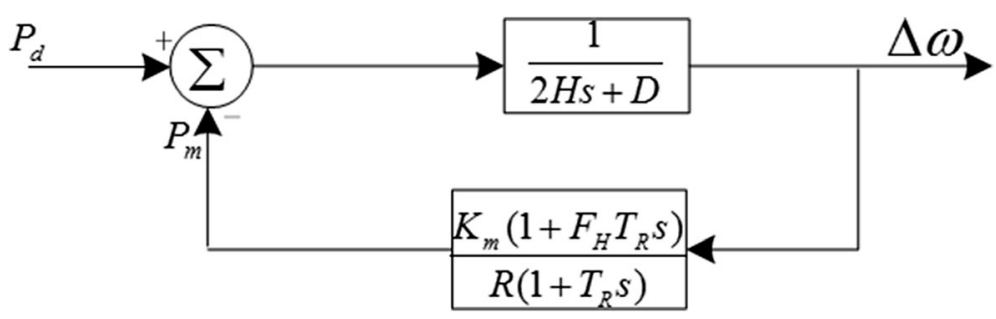

Fig. 3 Block diagram of SFR model 
of little interest or voltage can be held at desired levels, the active power-frequency dynamics can be decoupled from the reactive power-voltage dynamics for simplification. It makes active-power the only factor affecting frequency, and the reduced models introduced above are suitable to examine the key impact of active power on frequency.

\section{Architecture of software package incorporating reduced frequency response models}

\subsection{Framework of the software package}

The framework of the proposed software package is shown in Fig. 4 with the following modules:

(1) Model library: Models with great impact on the frequency dynamics should be modelled in the package. The models implemented in the package are discussed in the next section in detail.

(2)Data assembler: The data file in supported formats such as the IEEE and PSS/E data formats can be recognized and imported into the memory.

(3) Dynamic equivalence: The function of this module is to supply equivalence calculation for model parameters.

(4) Event library: From the information contained in event library, power system malfunctions or failures, such as generator tripping, load shedding/increasing and continuous loads variation can be set up.

(5) Frequency response calculation: The function of this module is to implement frequency response calculation. The reduced models, DFR, ASF, single machine and SFR model, are implemented in the package using the step-by-step integration method or analytical solution to calculate frequency response.

(6) Frequency security assessment: Security assessment module is implemented for advanced applications.
Details of the frequency security assessment module can be found in Section 3.4.

(7) Application program interfaces (API): The APIs are used to provide interface functions for advanced applications and secondary development.

\subsection{Model library}

The following models are implemented in the package with appropriate simplifications.

(a) Conventional generator: With decoupling of active power-frequency dynamics and reactive powervoltage dynamics, detailed generator models with damping windings are not required. In DFR model, generators are usually modelled as classical model with swing equation and constant internal voltage behind transient or sub-transient reactance. For ASF and single machine model, no transient or subtransient reactance is modelled. Only aggregated swing equations are kept in the ASF and single machine model.

(b) Turbine-governor: Turbine-governors provide the mechanical power for generators and are modelled in detail in the package. Typical turbine-governor models are only concerned with active power-frequency dynamics, with reactive power-voltage dynamics ignored. Thus, turbine-governor models can be reserved without simplification.

(c) Load: Considering only active power, two types of load models are implemented in the package: static load model considering frequency dependency and dynamic load model considering induction motors with active power-frequency dynamic response [21].

(d)HVDC: With more and more HVDC projects deployed, the control of HVDC should be modelled for frequency studies, which contains active power

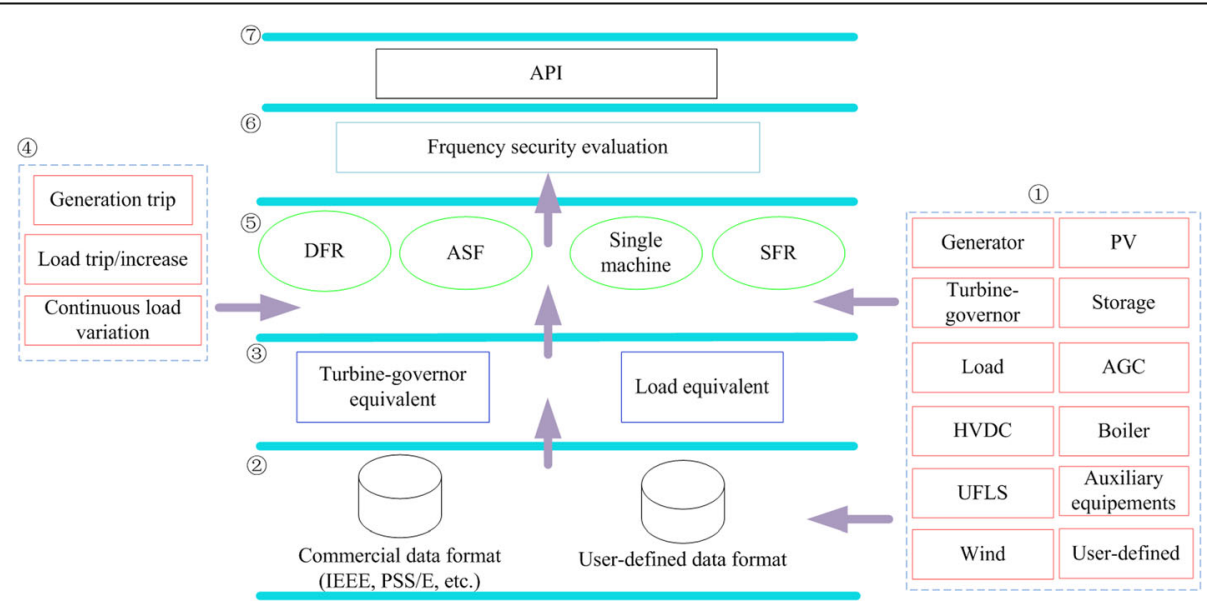

Fig. 4 Framework of the proposed package for frequency response calculation 
modulation, dead band of frequency deviation and active power order sub-modules.

(e) UFLS: The control strategies are important for power system frequency stability. Under-frequency load shedding is an important frequency control measure and is modelled in the package in detail.

(f) Wind generator: For wind farm participating into frequency regulation, electrical control is ignored whereas pitch control of the wind turbines is implemented for frequency regulation [22]. Meanwhile, in order to make the wind turbine dynamic process similar to conventional generating units, virtual inertia control can be considered.

(g) Photovoltaic (PV) generation and battery energy storage: With large-scale photovoltaic generation connected to power systems, its fluctuation determines the demands for battery allocation. The PV is usually modelled as negative loads and energy storage is modelled as loads with response to frequency changes.

(h)Protective relay: Generators are equipped with protective relaying devices and their malfunctions can cause serious active power disturbance and large frequency deviation. So the under/over frequency protective relays are included in this package.

(i) Boiler and automatic generation control (AGC): For the purpose of medium and long term simulation, boiler dynamics and AGC should be considered [23, 24].

(j) User-defined model: If other power system models are required, user-defined models can be added in the model library to extend the function of the package.

\subsection{Dynamic equivalence module}

There are many types of generators in power systems. For the single machine and SFR models, turbinegovernors and loads should be aggregated as single turbine-governor and load. Swing equation equivalence [25] and turbine-governor equivalence [26] are implemented in the dynamic equivalence module. For turbinegovernor equivalence, two sub-modules are developed for different purposes:

(a) Equivalence of the same type of turbine-governors. When aggregating several turbine-governors of the same type, model structure is retained and the parameters can be generally summed up with weights to give appropriate responses.

(b)Equivalence of different types of turbine-governors. When aggregating several turbine-governors of different types, appropriate model structure should be first selected and then parameters are optimized to match the overall dynamic characteristics.

There are many algorithms to deal with model parameter equivalence, such as particle swarm optimization method
(PSO) [27], dynamic aggregation [28, 29], weighted summation method and least square method [30,31]. Appropriate algorithms can be implemented for desired applications.

\subsection{Frequency security assessment}

The output results from the frequency response calculation module can be further analysed with the frequency security assessment module. This module provides transient frequency deviation security (TFDS) assessment and frequency security margin index based on two-element table [32]. According to the requirements of power system operation and control, frequency deviation constraints in extent and duration are given as twoelement table $\left[f_{c r} t_{c r}\right]$ where $f_{c r}$ is the deviation extent and $t_{c r}$ is the corresponding maximum duration. For a given frequency trajectory and two-element table $\left[f_{c n} t_{c r}\right]$, the frequency security index can be calculated by considering the cumulative effect of frequency deviation.

Frequency evaluation can be used for further frequency control decision-making. For example, for the setting of UFLS scheme, it can be used to check the feasibility of the scheme and provide guidance for how to optimize.

\subsection{API module}

The API module is divided into several sub-modules to realize different functional requirements. For example, data assembler APIs are used to read data, and equivalence APIs are used for dynamic parameter equivalence calculation. For security assessment and UFLS setting evaluation, APIs are also implemented for model extension and secondary development.

To improve the programmability, all APIs can be called via dynamic link library (DLL). The advantage of DLL over graphic user interface (GUI) is the freedom to prepare scripts for specific applications. Since almost all high-level languages support loading DLL, the package can be further implemented in other software to extend their functionality of frequency dynamics study.

\section{Case study}

A programmable and open software package with the framework proposed in Section 3 is implemented in this paper with $\mathrm{C}++$. Dynamic models of PSS/E are supported. The compiled DLL is called in Python modules named pydfr for DFR model, pyasf for ASF model, pysm for single machine model, pysfr for SFR model, pyeqv for model equivalence, and pyevl for transient frequency deviation security evaluation.

\subsection{Demonstration of APIs}

The following example shows how a simulation with Python codes calling APIs is performed. 


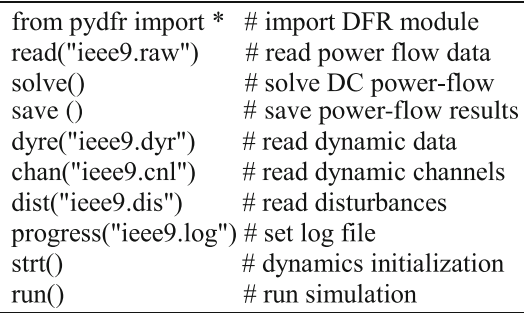

The API $\operatorname{read}()$ imports model data (including power flow and dynamic models) into the program. DC power flow is solved with API solve(), and power flow results are saved with API save(). Prior to running dynamic simulation, dynamic data should be imported with API dyre(), and the channels to be exported are set with API chan(). Events information can be imported by API dist(). If detailed action of the power system is expected, a dynamic action file can be set up with API progress(). Before running the simulation with API run(), API $\operatorname{strt}()$ is called to initialize the dynamic models. The simulation results are outputted automatically during simulation.

\subsection{Model equivalence}

To perform simulations with single machine and SFR models, model equivalence should be performed for multi-machine systems. In this paper, dynamic aggregation and weighted summation method are implemented for model equivalence calculation. The following codes show how an equivalence for the single machine model with typical steam turbine-governor model IEEEG1 is obtained.

\begin{tabular}{|ll}
\hline import pyeqv & \# model equivalence \\
import pydfr & \# DFR related module \\
pydfr.read("ieee9.raw") & \\
pydfr.solve() & \\
pydfr.dyre("ieee9.dyr") & \\
pyeqv.aggregate_st() & \\
pyeqv.aggregate_dt("IEEEG1") & \# aggregate the same type \\
\#aggregate different types
\end{tabular}

The data imported into the package by the pydfr module can be accessed by the pyeqv module. To get the proper equivalent model, the same type of turbine-governors are first aggregated by API aggregate_st(). The aggregated models can be used for ASF model. If single machine model is to be called, the API aggregate_dt() needs to be called to reduce turbine-governors of different types to a single model.

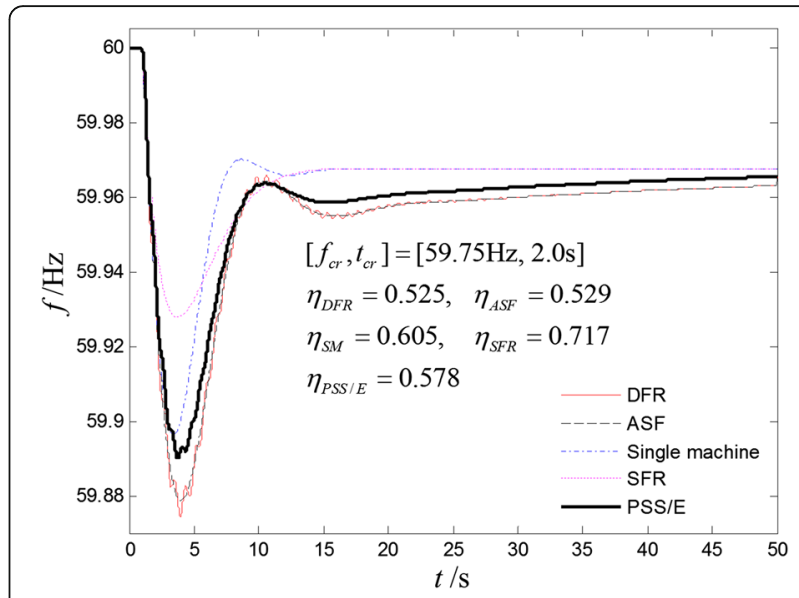

Fig. 5 Frequency response with increasing $8 \mathrm{MW}$ load in the IEEE 9-bus model

\subsection{Comparison of different models}

Frequency responses of the reduced models are examined in this section with the IEEE 9-bus model and a 1000 -bus model from China. Dynamic frequency of the two cases are shown in Figs. 5 and 6, respectively.

For the IEEE 9-bus model, PSS/E is adopted to perform the full time-domain simulation with complete models of generators, exciters, and turbine governors. Loads are modelled as $40 \%$ constant impedance load plus $60 \%$ constant power load with frequency dependency. In Fig. 5, the response captured by DFR deviates greater than that by PSS/E since the actual load in full time-domain simulation decrease slightly with the drop of voltage which is neglected in the DFR model. The DFR model generally reflects the overall frequency dynamics when voltage dynamics is neglected. For simplified models, the uniform

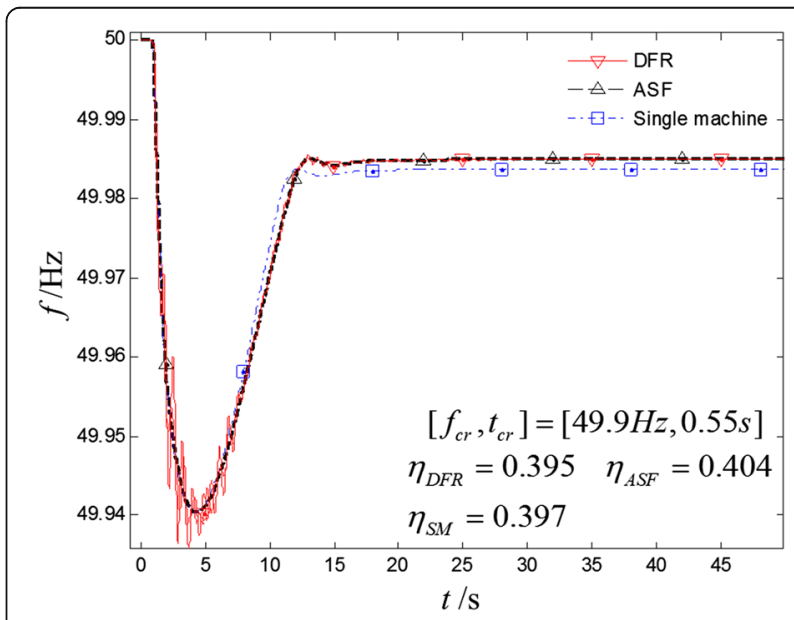

Fig. 6 Frequency response with tripping $300 \mathrm{MW}$ generation in the 1000-bus model 
frequency of the ASF model is almost the same as that of the DFR model, and both models can reflect frequency response process with good accuracy. The single machine model has similar tendency as the DFR and ASF models. However, the aggregation of the two steam turbines and one hydraulic turbine in the 9-bus model produces some errors, especially for the overshoot part around $8 \mathrm{~s}$. The linear SFR model gives different response from the nonlinear models where spinning reserve is limited. The values of the TFDS index $\eta$ are calculated by the security assessment module and are shown in Fig. 5 based on a twoelement table of $[59.75 \mathrm{~Hz}, 2.0 \mathrm{~s}]$.

For the 1000-bus model from China, similar conclusions can be drawn. Due to the lack of spinning reserve, the frequency response of the equivalent SFR model deviates greatly from the results of other models and is not illustrated in Fig. 6. The two-element table for the 1000bus model is $[49.9 \mathrm{~Hz}, 0.55 \mathrm{~s}]$.

To compare the computation efficiency between the reduced models, time consumption for the generator tipping event in Fig. 6 is compared with simulation time span of 50s. On a PC with $\mathrm{CPU}$ of $2.83 \mathrm{GHz}$, the simulation
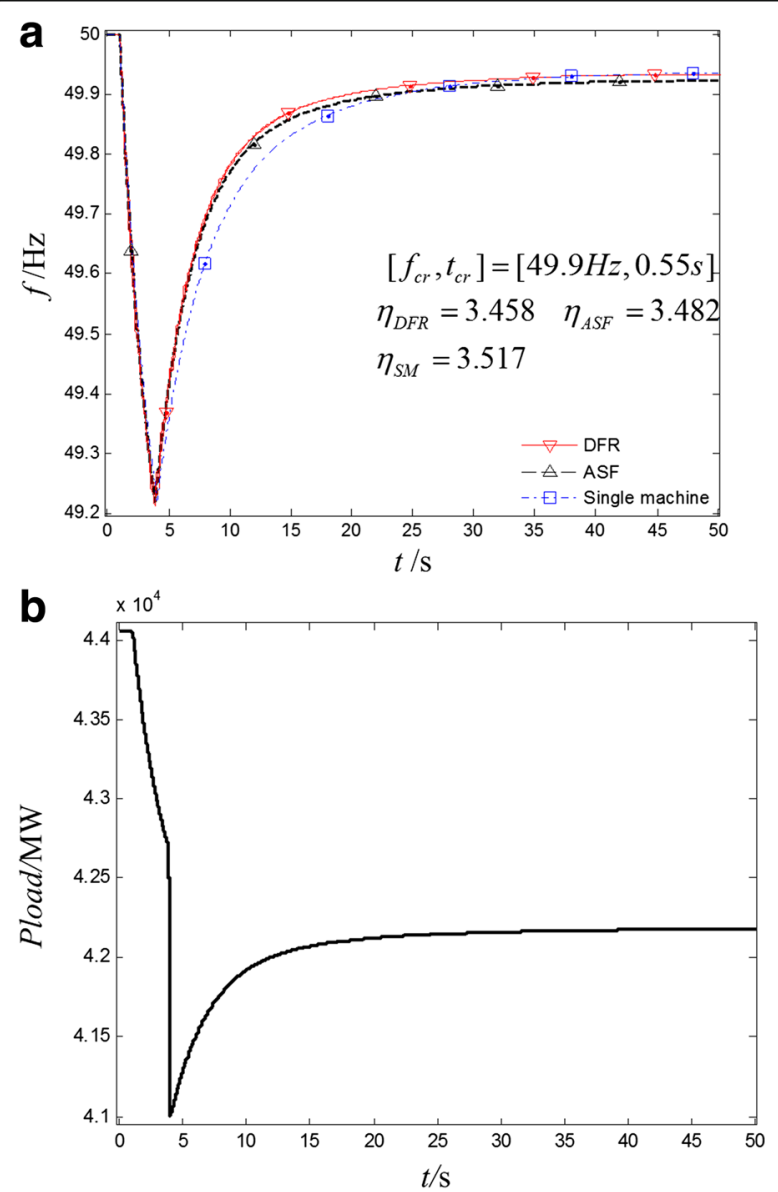

Fig. 7 Dynamics of tripping 5\% generation in the 1000-bus model with UFLS control. a Frequency response. b System total load time of the DFR model, ASF model, and single machine model are $3.259 \mathrm{~s}, 0.531 \mathrm{~s}$, and $<1 \mathrm{~ms}$, respectively. With the improvement of computational efficiency, the package is suitable for online frequency response analysis.

\subsection{UFLS control}

To demonstrate the applications of the package in UFLS control, a UFLS scheme is set up for the 1000-bus model. After tripping $5 \%$ of total generation, the first step of UFLS is activated when frequency drops beyond $49.25 \mathrm{~Hz}$ with time delay of $0.2 \mathrm{~s}$. Four percent of total load is shed to recover frequency. The dynamics of frequency and total load are shown in Fig. 7.

\subsection{Load variation}

With large scale integration of renewable generation, the fluctuation of renewables will lead to frequency variation. A load variation model is provided in this package to check the impact of variable loads and renewables (negative load) on frequency dynamics. On the NPCC 140-bus model, perturbation of renewable power is applied with
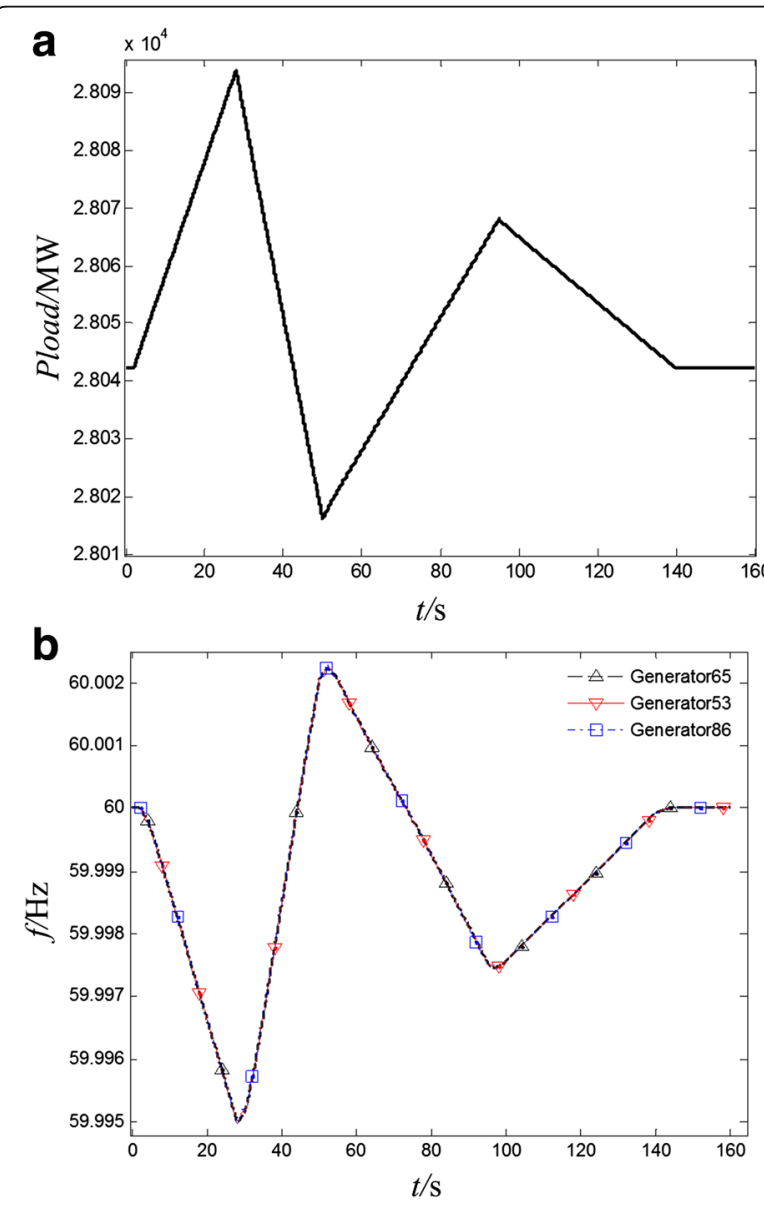

Fig. 8 Load variation example in NPCC model. a System total load. b Frequency response 
the load variation shown in Fig. 8 (a). The frequency response is shown in Fig. 8 (b).

\subsection{Advanced applications}

With the APIs provided, advanced applications can be conducted by calling the APIs with high-level languages. The following codes show the application of searching critical load shedding amount during a sudden generation trip to prevent further activation of UFLS. The general process of simulation and evaluation is re-defined in the new function sim_evl().

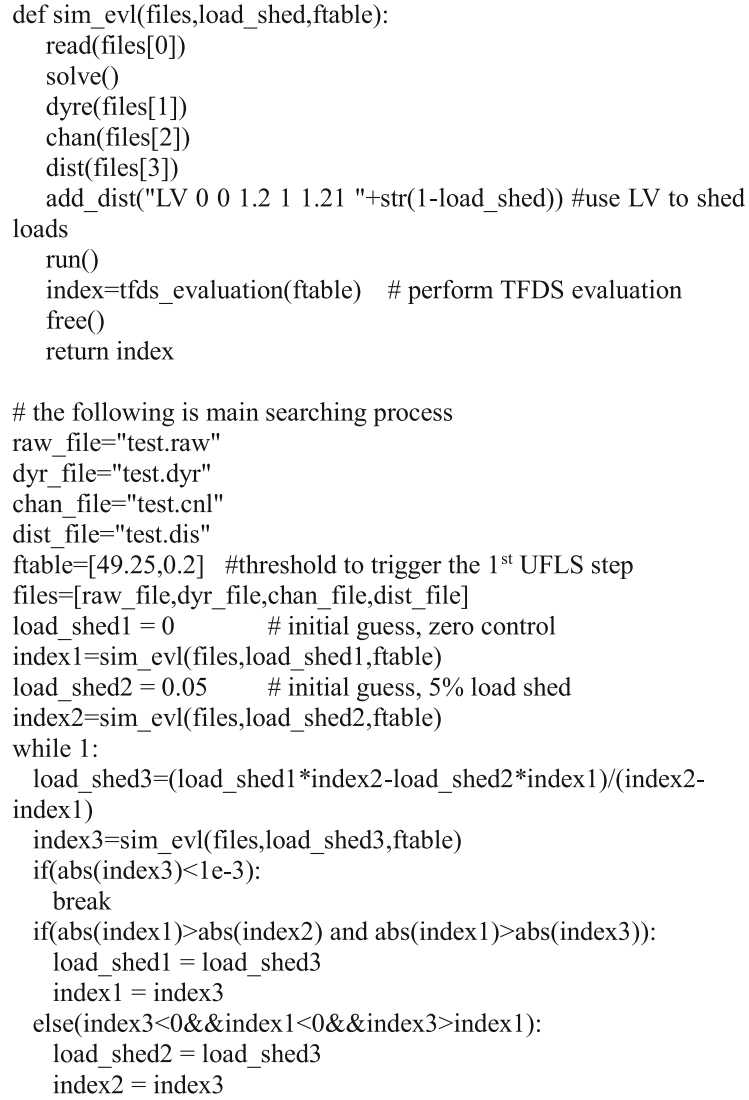

Figure 9 shows the iteration process to calculate the critical amount of load shedding. The two-element table of the 1st step of UFLS $([49.25 \mathrm{~Hz}, 0.2 \mathrm{~s}])$ is used to check the frequency security index. When the index is zero, the 1 st step of UFLS is critically activated. With the initial guess $(0 \%$ of iteration -1 , and $5 \%$ of iteration 0$)$, the searching progress converges after 6 iterations, and 1.71\% loads should be shed $0.2 \mathrm{~s}$ after the event to prevent the activation of the 1st step of UFLS. The percentage shown in Fig. 9 are the load shedding amount of each iteration.

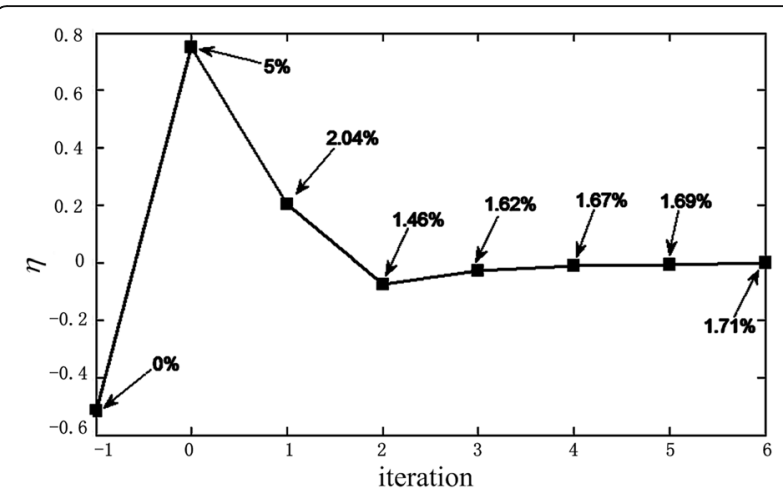

Fig. 9 Iteration of searching UFLS threshold

\section{Conclusion}

Development approach of a programmable and open software package for power system frequency response calculation is proposed in this paper where reduced frequency response models are incorporated. With the modularized framework, the package can be easily extended by adding new functions to the modules. APIs are provided in DLL to be called by other high-level languages. The programmability makes the package suitable for advanced applications and secondary development. An implementation of the proposed framework is introduced with support of PSS/E data formats. Simulations show that the software package is easy to use and APIs can be reorganized to perform simulations for specific purpose.

\section{Acknowledgement}

This work was supported by National Natural Science Foundation of China (No: 51477092)

\section{Authors' contributions}

$\mathrm{HZ}$ proposed the idea and helped to prepare and revise the draft. CL coded the simulation modules of DFR and ASF in C++, and prepared the draft. YX coded the aggregation module, evaluation module in $\mathrm{C}++$, and the Python modules. Most simulations were conducted by YX. HS helped to revise the draft and reorder the sequence of the draft. The model library part was revised based on HS's suggestion. All authors read and approved the final manuscript.

\section{About the authors}

Yuzheng Xie (1991-), M.E. candidate. Major in power system security, stability assessment and control.

Hengxu Zhang (1975-), Ph.D. and professor. Major in power system security and stability assessment, power system monitoring and numerical simulation. Changgang Li (1984-), Ph.D. and associate research fellow. Major in power system dynamic and control, and wide-area measurement and control. Huadong Sun (1975-), Ph.D and professor level senior engineer. Major in power system security assessment, stability control and numerical simulation.

\section{Competing interests}

In the past 5 years all the authors haven't received reimbursements, fees, funding, or salary from an organization that may in any way gain or lose financially from the publication of this comment, either now or in the future.

All the authors don't hold any stocks or shares in an organization that may in any way gain or lose financially from the publication of this comment, either now or in the future.

All the authors don't hold and aren't currently applying for any patents relating to the content of the comment. All the authors don't haven't received 
reimbursements, fees, funding, or salary from an organization that holds or has applied for patents relating to the content of the comment.

All the authors don't don't have any other financial competing interests. There aren't any non-financial competing interests (political, personal, religious, ideological, academic, intellectual, commercial or any other) to declare in relation to this comment

\section{Author details}

'Key Laboratory of Power System Intelligent Dispatch and Control of the Ministry of Education (Shandong University), 17923 Jingshi Road, Jinan, Shandong 250061, China. ${ }^{2}$ China Electric Power Research Institute, 15 Xiaoying East Road, Qinghe, Beijing 100192, China.

Received: 29 December 2016 Accepted: 20 March 2017

Published online: 08 May 2017

\section{References}

1. Kundur, P., Paserba, J., Ajjarapu, V., et al. (2004). Definition and classification of power system stability. IEEE Transactions on Power Systems, 19(3), 1387-1401.

2. Gu, W., Liu, W., Zhu, J. P., et al. (2014). Adaptive Decentralized UnderFrequency Load Shedding for Islanded Smart Distribution Networks. IEEE Transactions on Sustainable Energy, 5(3), 886-895.

3. Larsson M. (2005) An adaptive predictive approach to emergency frequency control in electric power systems, 44th IEEE Conference on Decision Control, pp. 4434-4439.

4. Ahsan, M. Q., Chowdhury, A. H., Ahmed, S. S., et al. (2012). Technique to Develop Auto Load Shedding and Islanding Scheme to Prevent Power System Blackout. IEEE Transactions on Power Systems, 27(1), 198-205.

5. Wang, S. P., Chen, A., Liu, C. W., et al. (2015). Efficient Splitting Simulation for Blackout Analysis. IEEE Transactions on Power Systems, 30(4), 1775-1783.

6. Medina, D. R., Rappold, E., Sanchez, O., et al. (2016). Fast Assessment of Frequency Response of Cold Load Pickup in Power System Restoration. IEEE Transactions on Power Systems, 31(4), 3249-3256.

7. Henneaux, P., Labeau, P. E., \& Maun, J. C. (2013). Blackout Probabilistic Risk Assessment and Thermal Effects: Impacts of Changes in Generation. IEEE Transactions on Power Systems, 28(4), 4722-4731.

8. Gurusinghe, D. R., Rajapakse, A. D., \& Narendra, K. (2014). Testing and Enhancement of the Dynamic Performance of a Phasor Measurement Unit. IEEE Transactions on Power Delivery, 29(4), 1551-1560.

9. Lin, Z. Z., Xia, T., Ye, Y. Z., et al. (2013). Application of wide area measurement systems to islanding detection of bulk power systems. IEEE Transactions on Power Systems, 28(2), 2006-2015.

10. Liu, Y., Zhan, L. W., Zhang, Y., et al. (2016). Wide-Area-Measurement System Development at the Distribution Level: An FNET/GridEye Example. IEEE Transactions on Power Delivery, 31(2), 721-731.

11. Jin Z. S., Zhang H. X. Li C. G. (2015) WAMS Light and Its Deployment in China', the 5th International Conference on Electric Utility Deregulation and Restructuring and Power Technologies (DRPT), Changsha, China, pp. 1373-1376

12. Zhang, H. X., Shi, F., Liu, Y. T., et al. (2016). Adaptive Online Disturbance Location Considering Anisotropy of Frequency Propagation Speeds. IEEE Transactions on Power Systems, 31(2), 931-941.

13. Chan, M. L., Dunlop, R. D., \& Schweppe, F. (1972). Dynamic equivalents for average system frequency behaviour following major disturbances. IEEE Transactions on Power Apparatus and Systems, PAS-91(4), 1637-1642.

14. Anderson, P. M., \& Mirheydar, M. (1990). A low-order system frequency response model. IEEE Transactions on Power Systems, 5(3), 720-729.

15. Stott, B., Jardim, J., \& Alsac, O. (2009). DC Power Flow Revisited. IEEE Transactions on Power Systems, 24(3), 1290-1300.

16. Wang K., Huang H., Zang C. (2013) 'Research on Time-Sharing ZIP Load Modeling Based on Linear BP Network', The 5th Intelligent Human-Machine Systems and Cybernetics Conference (IHMSC), Hangzhou, China, pp. 37-41

17. Ersdal, A. M., Imsland, L., \& Uhlen, K. (2016). Model Predictive LoadFrequency Control. IEEE Transactions on Power Systems, 31(1), 777-785.

18. Yousef, H. A., AL-Kharusi, K., Albadi, M. H., et al. (2014). Load Frequency Control of a Multi-Area Power System: An Adaptive Fuzzy Logic Approach. IEEF Transactions on Power Systems, 29(4), 1822-1830.

19. Anderson, P. M., \& Mirheydar, M. (1992). An adaptive method for setting under-frequency load shedding relays. IEEE Transactions on Power Systems, $7(2), 647-655$
20. Denis, L. (2006). A general-order system frequency response model incorporating load shedding: analytic modeling and applications. IEEE Transactions on Power Systems, 21(2), 709-717.

21. Aree, P., \& Acha, E. (2011). Power flow initialisation of dynamic studies with induction motor loads. IET Generation, Transmission \& Distribution, 5(4), 417-424.

22. Chang-Chien, L. R., Lin, W. T., \& Yin, Y. C. (2011). Enhancing frequency response control by DFIGs in the high wind penetrated power systems. IEEE Transactions on Power Systems, 26(2), 710-718.

23. Xie Y. Z., Zhang H. X., Sun H. D., et al. (2015) Frequency response model considering large frequency deviation, Industrial Instrumentation and Control Conference (ICIC), Pune, India, pp. 940-943.

24. De Mello, F. P. (1991). Boiler models for system dynamic performance studies. IEEE Transactions on Power Systems, 6(1), 66-74.

25. Zhang B. Z, Zhang Y., Lin L. X., et al. (2012) Study on Two Dynamic Aggregation Algorithms of Coherent Generators, the 4th IEEE Computational Intelligence and Communication Networks Conference (CICN), Mathura, India, pp. 676-680

26. Ourari, M. L., Dessaint, L. A., \& Do, V. Q. (2006). Dynamic equivalent modeling of large power systems using structure preservation technique. IEEE Transactions on Power Systems, 21(3), 1284-1295.

27. Voumvoulakis, E. M., \& Hatziargyriou, N. D. (2010). A Particle Swarm Optimization Method for Power System Dynamic Security Control. IEEE Transactions on Power Systems, 25(2), 1032-1041.

28. Ju, P., Ni, L. Q., \& Wu, F. (2004). Dynamic equivalents of power systems with online measurements. Part 1: Theory. IEE Proceedings-Generation, Transmission and Distribution, 151(2), 175-178.

29. Ju, P., Li, F., Yang, N. G., et al. (2004). Dynamic equivalents of power systems with online measurements Part 2: Applications. IEE Proceedings-Generation, Transmission and Distribution, 151(2), 179-182

30. Wan, J., \& Miu, K. N. (2003). Weighted least squares methods for load estimation in distribution networks. IEEE Transactions on Power Systems, 18(4), 1338-1345.

31. D'Antona, G. (2003). The full least-squares method. IEEE Transactions on Instrumentation and Measurement, 52(1), 189-196.

32. Zhang, H. X., Li, C. G., \& Liu, Y. T. (2015). Quantitative frequency security assessment method considering cumulative effect and its applications in frequency control. International Journal of Electrical Power \& Energy Systems, 65(65), 12-20

\section{Submit your manuscript to a SpringerOpen ${ }^{\odot}$ journal and benefit from:}

- Convenient online submission

- Rigorous peer review

- Immediate publication on acceptance

- Open access: articles freely available online

- High visibility within the field

- Retaining the copyright to your article

Submit your next manuscript at $>$ springeropen.com 